\title{
Moderators of changes in smoking, drinking and quitting behaviour associated with the first COVID-19 lockdown in England
}

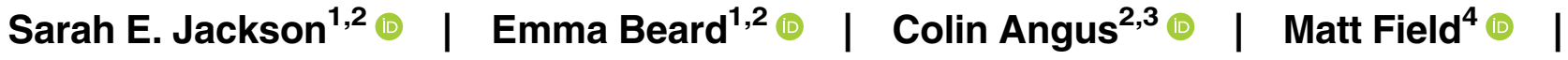 \\ Jamie Brown ${ }^{1,2}$ ()
}

${ }^{1}$ Department of Behavioural Science and Health, University College London, London, UK

${ }^{2}$ SPECTRUM Consortium, UK

${ }^{3}$ School of Health and Related Research, University of Sheffield, UK

${ }^{4}$ Department of Psychology, University of Sheffield, UK

\section{Correspondence}

Sarah Jackson, Department of Behavioural Science and Health, University College London, 1-19 Torrington Place, London WC1E 7HB, UK.

Email: s.e.jackson@ucl.ac.uk

Funding information

Cancer Research UK, Grant/Award Number: C1417/A22962

\begin{abstract}
Aim: To estimate changes in smoking, drinking and quitting behaviour from before to during the first COVID-19 lockdown in England, and whether changes differed by age, sex or social grade.

Design: Representative cross-sectional surveys of adults, collected monthly between August 2018 and July 2020.

Setting: England.

Participants: A total of 36980 adults ( $\geq 18$ years).

Measurements: Independent variables were survey month (pre-lockdown: August-February versus lockdown months: April-July) and year (pandemic: 2019/20 versus comparator: 2018/19). Smoking outcomes were smoking prevalence, cessation, quit attempts, quit success and use of evidence-based or remote cessation support. Drinking outcomes were high-risk drinking prevalence, alcohol reduction attempts and use of evidence-based or remote support. Moderators were age, sex and occupational social grade $(A B C 1=$ more advantaged/C2DE = less advantaged).

Findings: Relative to changes during the same time period in $2018 / 19$, lockdown was associated with significant increases in smoking prevalence [ $+24.7 \%$ in $2019 / 20$ versus $0.0 \%$ in $2018 / 19$, adjusted odds ratio (aOR) $=1.35,95 \%$ confidence interval $(\mathrm{Cl})=1.12-1.63]$ and quit attempts $(+39.9$ versus $-22.2 \%$, aOR $=2.48,95 \% \mathrm{Cl}=1.76-3.50$ ) among $18-34$-year-olds, but not older groups. Increases in cessation $(+156.4$ versus $-12.5 \%$, $\mathrm{aOR}=3.08,95 \% \mathrm{Cl}=1.86-5.09)$ and the success rate of quit attempts ( +99.2 versus $+0.8 \%$, aOR $=2.29,95 \% \mathrm{Cl}=1.31-3.98)$ were also observed, and did not differ significantly by age, sex or social grade. Lockdown was associated with a significant increase in high-risk drinking prevalence among all socio-demographic groups $(+39.5$ versus $-7.8 \%, \quad \mathrm{aOR}=1.80,95 \%$ $\mathrm{Cl}=1.64-1.98)$, with particularly high increases among women $(\mathrm{aOR}=2.17$, $95 \% \mathrm{Cl}=1.87-2.53)$ and social grades $\mathrm{C} 2 \mathrm{DE}(\mathrm{aOR}=2.34,95 \% \mathrm{Cl}=2.00-$ 2.74). Alcohol reduction attempts increased significantly among high-risk drinkers from social grades $\mathrm{ABC} 1(\mathrm{aOR}=2.31,95 \% \mathrm{Cl}=1.78-3.00)$ but not $\mathrm{C} 2 \mathrm{DE}(\mathrm{aOR}=1.25,95 \% \mathrm{Cl}=0.83-1.88)$. There were few significant changes in use of support for smoking cessation or alcohol reduction, although samples were small.

Conclusions: In England, the first COVID-19 lockdown was associated with increased smoking prevalence among younger adults and increased high-risk drinking prevalence among all adults. Smoking cessation activity also
\end{abstract}

This is an open access article under the terms of the Creative Commons Attribution License, which permits use, distribution and reproduction in any medium, provided the original work is properly cited.

(C) 2021 The Authors. Addiction published by John Wiley \& Sons Ltd on behalf of Society for the Study of Addiction. 
increased: more younger smokers made quit attempts during lockdown and more smokers quit successfully. Socio-economic disparities in drinking behaviour were evident: high-risk drinking increased by more among women and those from less advantaged social grades (C2DE), but the rate of reduction attempts increased only among the more advantaged social grades $(A B C 1)$.

\section{KEYWORDS}

Alcohol, alcohol reduction, COVID-19, drinking, quit attempts, SARS-CoV-2, smoking, smoking cessation

\section{INTRODUCTION}

In order to suppress transmission of the SARS-CoV-2 virus, governments around the world have implemented guidelines and legislation to restrict social interaction, including advice to stay at home ('lockdown' restrictions). These restrictions may have influenced smoking, drinking and quitting behaviours in various ways. Some people may have used tobacco or alcohol as a means of coping with increased stress or boredom. Others may have taken the opportunity to quit smoking or drink less while daily routines are disrupted and social activity is reduced. Given the different social, financial and mental health impacts of lockdown on different sociodemographic groups [1-4], it is plausible that changes in smoking and drinking have varied according to age, sex or socio-economic position. Understanding howand among which groups-smoking, drinking and quitting behaviours have changed in response to COVID-19 lockdown restrictions is essential for building a clear picture of their public health impact and targeting messaging and support services.

The COVID-19 pandemic began to seriously affect the United Kingdom in March 2020. The first UK death from COVID-19 was recorded on 2 March and the number of confirmed cases increased rapidly from 47 at the end of February to a total of more than 38000 by the end of March [5], with many more probably unrecorded with estimates of approximately 114000 a day [6]. The UK Coronavirus Action Plan [7] was published on 3 March, followed by government advice to practice social distancing on 16 March and a lockdown with behavioural restrictions enforceable by law on 23 March [8]. The British public were instructed that they must stay at home, except for certain 'very limited purposes', which included shopping for essential items (such as food or medicine); one form of outdoor exercise each day (such as running, walking or cycling), either alone or with others who live in the same household; for any medical need, or to provide care to a vulnerable person; and to travel to and from work where this was 'absolutely necessary' and the work in question could not be done from home [9]. All non-essential shops, libraries, places of worship, playgrounds and outdoor gyms were closed. Schools were closed for all in-person teaching, except for children of key workers and children considered vulnerable.

The Smoking and Alcohol Toolkit Studies [10,11] are ongoing monthly cross-sectional surveys of the adult population in England that have been designed to provide insights into population-wide influences on smoking and drinking behaviour. These surveys predate the COVID-19 pandemic, providing the opportunity to evaluate the impact of lockdown restrictions on smoking and drinking behaviour.

Using the first monthly data collected after the first COVID-19 lockdown was implemented in England, we recently published an evaluation of population-level changes in smoking, quit attempts, drinking and alcohol reduction attempts from before (April 2019February 2020) to during (April 2020) lockdown [12]. This revealed some positive changes in smoking and drinking outcomes during lockdown: smokers and high-risk drinkers were more likely than before lockdown to report trying to quit (39.6 versus 29.1\%) or reduce their alcohol consumption (28.5 versus $15.3 \%$ ), and the rate of smoking cessation doubled. However, high-risk drinking prevalence increased (38.3 versus $25.1 \%$ ) during lockdown and there was some evidence that use of evidence-based support for alcohol reduction by high-risk drinkers decreased, with no compensatory increase in use of remote support options.

With only 1 month of post-lockdown data available at the time this analysis was undertaken, we were unable to establish whether these changes were sustained over time. We also lacked sufficient sample size in the post-lockdown period to explore potential moderation of these changes by key variables such as age, sex or social grade. Understanding more about how these patterns of behaviour changed (or persisted) during the lockdown and how they differed between groups is important for informing health communications and policy decisions regarding provision and targeting of support for smoking cessation and alcohol reduction.

With additional data now available, this study therefore aimed to update and extend our original analysis of changes in smoking, drinking, quitting and alcohol 
reduction attempts during the first COVID-19 lockdown in England (April-July 2020). We aimed to (i) establish whether the immediate changes following the outbreak of COVID-19 compared with pre-lockdown that we observed in our original analysis persisted throughout the entire 4-month lockdown and (ii) examine the extent to which these changes were moderated by age, sex and social grade. Specifically, we addressed the following research questions:

1. Among adults in England, have changes in the prevalence of smoking or high-risk drinking following the outbreak of COVID-19 persisted and, if so, to what extent?

2. Among past-year smokers and after adjusting for socio-demographic characteristics and nicotine dependence, have changes in the prevalence of cessation following the outbreak of COVID-19 persisted?

3. Among past-year smokers and after adjusting for socio-demographic characteristics, have the changes in the prevalence of quit attempts following the outbreak of COVID-19 persisted?

4. Among past-year smokers attempting to quit, and after adjusting for socio-demographic characteristics and nicotine dependence, have changes in the rate of quit success or the prevalence of the use of cessation support following the outbreak of COVID-19 persisted?

5. Among high-risk drinkers and after adjusting for socio-demographic characteristics, have changes in the prevalence of alcohol reduction attempts following the outbreak of COVID-19 persisted?

6. Among high-risk drinkers attempting to reduce their alcohol consumption, and after adjusting for sociodemographic characteristics and alcohol dependence, have changes in the prevalence in the use of support for alcohol reduction following the outbreak of COVID-19 persisted?

7. Are the above changes moderated by age, sex or occupational social grade (as an index of socioeconomic position)?

\section{METHOD}

\section{Design}

Data were drawn from the ongoing Smoking and Alcohol Toolkit Studies [10,11]. The study uses a form of random location sampling to select a new sample of approximately 1700 adults each month. Interviews are performed with one household member until quotas based on factors influencing the probability of being at home (e.g. gender, age, working status) are fulfilled. Comparisons with sales data and other national surveys show that the Toolkit studies recruit a representative sample of the population in England with regard to key demographic variables, smoking prevalence and cigarette consumption [10,13]. Data are usually collected monthly through face-to-face computer-assisted interviews. However, social distancing restrictions under the COVID-19 pandemic meant that no data were collected in March 2020 and data from April 2020 onwards were collected via telephone, and the lower age bound for participation was increased from 16 to 18 years due to changes in consenting procedures. The telephone-based data collection relied upon the same combination of random location and quota sampling, and weighting approach as the face-to-face interviews and previous diagnostic analyses conducted on the first month of telephone data indicate good comparability between the two data collection modalities [12].

Lockdown restrictions in the United Kingdom were introduced on 23 March 2020 and were subsequently eased on 4 July. For the present study, we used data from respondents to the survey in the period from August 2018 to July 2020. Because the sample was restricted to people aged $\geq 18$ years when data collection switched from face-to-face to telephone interviews (due to different consenting procedures), we excluded any participants aged 16-17 recruited before lockdown for consistency.

\section{Measures}

\section{Exposure: timing of lockdown}

Our analyses focused upon tests of the interaction between survey month and year in order to establish whether any changes associated with the timing of the COVID-19 lockdown in March 2020 were over and above usual seasonal variation in our outcomes of interest. For our primary analyses, survey month was coded 0 (i.e. before lockdown) for respondents to the survey in August to February and 1 (i.e. during lockdown) for respondents to the survey in April to July. Survey year was coded 0 for respondents to the survey from August 2018 to July 2019 (i.e. comparator year) and 1 for respondents to the survey from August 2019 to July 2020 (i.e. pandemic year). The length of the prelockdown period was reduced from our original analysis (which included data from May rather than August [12]) to avoid overlap between the post-lockdown period in the comparator year and pre-lockdown period in the pandemic year.

Because lockdown restrictions in England were eased on 4 July 2020 and it was probable that much of the July data would have been obtained after this date, we conducted sensitivity analyses excluding July data from the lockdown period. 


\section{Outcomes}

Among all adults, we assessed the prevalence of current smoking and high-risk drinking [defined by an Alcohol Use Disorders Identification Test-consumption (AUDIT-C)] score $\geq 5$ [14].

Among past-year smokers, we assessed cessation and quit attempts in the past year. Among past-year smokers who reported a quit attempt, we assessed quit success, use of evidence-based support [defined as face-to-face behavioural support, prescription medication-varenicline, bupropion or nicotine replacement therapy (NRT), e-cigarettes or NRT obtained over the counter] and use of remote support [defined as telephone support, a website or an application (app)].

Among high-risk drinkers, we assessed alcohol reduction attempts in the past year. Among high-risk drinkers who reported a reduction attempt, we assessed use of evidence-based support (defined as face-to-face behavioural support or prescription medication) and use of remote support (defined as telephone support, a website or an app).

See Supporting information, File S1 for full details of the measures used to assess each outcome variable.

\section{Covariates}

Socio-demographic characteristics included age, sex, social grade and region in England. Age was categorized as 18-24, 25-34, 35-44, 45-54, 55-64 and $\geq 65$ years (those aged 16 or 17 who responded before April 2020 were excluded to match the age range of the sample collected during lockdown). For tests of interactions with age, we reduced the number of categories, coding age groups as 18-34, 35-64 and $\geq 65$ years. Social grade was categorized as $A B C 1$ (which includes managerial, professional and intermediate occupations) versus C2DE (which includes small employers and own-account workers, lower supervisory and technical occupations, and semi-routine and routine occupations, never workers and long-term unemployed). Region in England was categorized as London, South, Central and North.

We also included measures of nicotine and alcohol dependence. Nicotine dependence was assessed with the heaviness of smoking index [15], an index derived from the number of cigarettes smoked per day and time to the first cigarette of the day. Scores range from 0 (low dependence) to 6 (high dependence). Alcohol dependence was assessed with the (full, 10-item) AUDIT [14]. Scores range from 0-40, with 0-7 indicating low-risk consumption, 8-19 indicating hazardous or harmful consumption and $\geq 20$ indicating risk of alcohol dependence (moderate-severe alcohol use disorder).

\section{Statistical analysis}

The protocol and analysis plan was pre-registered on Open Science Framework (https://osf.io/zf6vp/); research questions were exploratory in nature. Data were analysed using SPSS version 24. Data were weighted to match the English population profile on age, social grade, region, tenure, ethnicity and working status within sex. The dimensions are derived monthly from a combination of the English 2011 census, Office for National Statistics mid-year estimates and an annual random probability survey conducted for the National Readership Survey. Missing cases were excluded on a per-analysis basis. Variables with the highest number of missing cases were heaviness of smoking index $(6.8 \%$ missing among past-year smokers), alcohol reduction attempts $(2.9 \%$ among high-risk drinkers), smoking quit attempts $(2.5 \%$ among past-year smokers) and high-risk drinking $(0.7 \%$ among all participants), with all other variables having $<0.5 \%$ missing data. We applied a false discovery rate correction [16] to all $P$-values using an on-line calculator (https://www.sdmproject.com/utilities/?show=FDR) to adjust for multiple comparisons.

For each outcome, we analyzed the prevalence by survey month [before [August-February) versus after (April-July)] and year (pandemic [2019/20] versus comparator [2018/19]) and constructed a logistic regression model testing the month $\times$ year interaction to test whether observed differences between months before and during lockdown were larger in the pandemic year than the comparator year. These models adjusted for time trends within years (i.e. from August $=1$ to July$=12$ ) and throughout the entire analyzed period (i.e. from August $2018=1$ to July $2020=24$ ). Estimates of smoking and high-risk drinking prevalence did not have any additional adjustment, as they were weighted on important dimensions to match the population in England. Analyses of quit/reduction attempts were adjusted for age, sex, social grade and region (to take account of small differences in the make-up of the subgroups being analyzed). Analyses of smoking cessation, quit success and use of support were adjusted for socio-demographic characteristics and level of dependence (because more dependent smokers tend to be less likely to quit and more dependent smokers/drinkers tend to be more likely to use support).

In order to test for moderation of associations, we ran a series of models for each outcome (fully adjusted for any relevant covariates, as described in the previous paragraph) in which the three-way interactions between the survey month (before versus during lockdown), year (pandemic versus comparator) and (i) age (18-34, 35-59 and $\geq 60$ years), (ii) sex (male versus female) and (iii) social grade (ABC1 versus C2DE) were added. Each of the three-way interactions were 
tested in separate models. Where there was evidence of moderation, we ran stratified analyses in which the two-way interaction between survey month and year was tested separately for each level of the moderating variable (i.e. separately by age group, sex or social grade) to provide more information as to the nature of the differences between groups.

To evaluate the impact of the change in modality of data collection from face-to-face (up to February 2020) to telephone (from April 2020), we replicated the diagnostic analyses we undertook in our original paper to check on the representativeness of the sample or comparability of data from wave to wave now that a large sample has been recruited via telephone. Results were very similar to those we reported in our previous paper (Supporting information, File S2), and suggest that it is reasonable to compare data from before and after the lockdown, despite the change in data collection method.

\section{ETHICS APPROVAL AND CONSENT TO PARTICIPATE}

Ethical approval for the STS/ATS was granted originally by the UCL Ethics Committee (ID 0498/001). The data are not collected by UCL and are anonymized when received by UCL.

\section{RESULTS}

\section{Sample characteristics}

A total of 36980 adults aged $\geq 18$ years participated in the Smoking and Alcohol Toolkit Study between August 2018 and July 2020 [mean $=1681$, standard deviation $(\mathrm{SD})=30.3$ per month]. Socio-demographic characteristics of the sample by survey year (pandemic: 2019/20 versus comparator: 2018/19) and month (during lockdown: April-July versus before lockdown: August-February) are shown in Table 1.

\section{Associations of lockdown with changes in smoking outcomes}

Table 2 shows changes in the prevalence of current smoking, cessation, quit attempts, quit success and use of cessation support from before (August-February 2019/20) to during the COVID-19 lockdown (April-July 2020) relative to changes in these variables over the previous year (August-February 2018/19 to April-July 2019). Figure 1 shows the monthly prevalence of smoking outcomes throughout the entire study period. Supporting information, File S3, Table 1 summarizes tests of moderation of associations between the
COVID-19 lockdown and changes in smoking outcomes by age, sex and social grade.

Among all adults, smoking prevalence was fairly stable from before to during lockdown $(+0.3 \%$ from August-February 2019/20 to April-July 2020). However, a significant interaction with age [adjusted odds ratio $(\mathrm{aOR})=0.60,95 \%$ confidence interval $(\mathrm{Cl})$ $=0.43-0.83$ for $\geq 60$ versus 18-34] revealed an increase in smoking prevalence among 18-34-yearolds $(+24.7 \%)$ that was significantly greater than changes in this group over the same time-period in $2018 / 19$ in the absence of lockdown restrictions $(0.0 \%$ change).

Among past-year smokers, lockdown was associated with significant increases in the rate of cessation $(+156.4 \%)$ and quit attempts $(+39.9 \%)$, over and above changes over the same time-period in 2018/19 $(-12.5 \%$ and $+0.8 \%$, respectively). Changes in cessation did not differ significantly by smokers' age, sex or social grade, but the change in the prevalence of quit attempts was moderated by age $(\mathrm{aOR}=0.37$, $95 \% \quad \mathrm{Cl}=0.23-0.61) \quad$ and $\quad \mathrm{aOR}=0.38 \quad(95 \%$ $\mathrm{Cl}=0.19-0.76$ ) for $35-59$ and $\geq 60$ versus $18-34$. Among 18-34 year-old smokers, there was a substantial increase in quit attempts $(+39.9 \%)$ contrasted against a decline during the same period in 2018/19 $(-22.2 \%)$. By contrast, changes among 35-59-yearolds and $\geq 60$-year-olds from before to during lockdown were similar to those observed during the same timeperiod in 2018/19.

Among past-year smokers who made a quit attempt, lockdown was associated with a significant increase in the success rate of quit attempts (+99.2\%), which had previously been stable during the same time-period in 2018/19 (+0.8\%). Changes in quit success did not differ significantly by smokers' age, sex or social grade. There was no significant association between lockdown and use of evidence-based or remote support for smoking cessation, although the relatively small sample sizes should be noted.

\section{Associations of lockdown with changes in drinking outcomes}

Table 3 shows changes in the prevalence of high-risk drinking, alcohol reduction attempts and use of support for alcohol reduction from before (August-February 2019/20) to during the COVID-19 lockdown (April-July 2020) relative to changes in these variables during the previous year (August-February 2018/19 to April-July 2019). Figure 2 shows the monthly prevalence of drinking outcomes throughout the entire study period. Supporting information, File S3, Table 2 summarizes tests of moderation of associations between the COVID-19 lockdown and changes in drinking outcomes by age, sex and social grade. 
T A B LE 1 Sample characteristics by survey year and month.

\begin{tabular}{|c|c|c|c|c|}
\hline \multirow[b]{2}{*}{$n$} & \multicolumn{2}{|c|}{ Comparator year (2018/19) } & \multicolumn{2}{|l|}{ Pandemic year (2019/20) } \\
\hline & $\begin{array}{l}\text { August 2018- } \\
\text { February 2019 } \\
11836\end{array}$ & $\begin{array}{l}\text { April-July } 2019 \\
6593\end{array}$ & $\begin{array}{l}\text { August 2019-February } 2020 \\
\text { (before lockdown) } \\
11897\end{array}$ & $\begin{array}{l}\text { April-July } 2020 \\
\text { (during lockdown) } \\
6655\end{array}$ \\
\hline \multicolumn{5}{|c|}{ Age, years, \% (n) } \\
\hline $18-24$ & $12.9(1526)$ & $12.4(817)$ & $12.7(1509)$ & $10.9(726)$ \\
\hline $45-54$ & $17.4(2054)$ & $17.2(1134)$ & $17.1(2038)$ & $17.2(1142)$ \\
\hline $55-64$ & $14.4(1701)$ & $14.6(964)$ & $14.6(1739)$ & $15.2(1013)$ \\
\hline$\geq 65$ & $22.4(2654)$ & $22.6(1489)$ & $22.7(2702)$ & $23.3(1552)$ \\
\hline \multicolumn{5}{|l|}{ Sex, \% (n) } \\
\hline $\mathrm{ABC} 1$ & $55.9(6618)$ & $55.6(3664)$ & $55.5(6602)$ & $53.4(3555)$ \\
\hline C2DE & $44.1(5218)$ & 44.4 (2929) & $44.5(5295)$ & 44.3 (2949) \\
\hline Missing & $0(0)$ & $0(0)$ & $0(0)$ & $2.3(151)$ \\
\hline \multicolumn{5}{|c|}{ Region in England, \% ( $n$ ) } \\
\hline London & $15.6(1847)$ & $15.7(1036)$ & $15.5(1842)$ & $15.4(1023)$ \\
\hline South & 26.4 (3122) & $26.3(1731)$ & $26.4(3146)$ & $26.6(1769)$ \\
\hline Central & $30.3(3588)$ & $30.1(1983)$ & $30.1(3575)$ & $30.4(2022)$ \\
\hline North & $27.7(3280)$ & $28.0(1843)$ & $28.0(3333)$ & $27.7(1841)$ \\
\hline
\end{tabular}

All data are weighted to match the adult population in England on age, social grade, region, tenure, ethnicity and working status within sex. In some cases, subgroup numbers do not sum to the total number due to rounding.

Among all adults, the COVID-19 lockdown was associated with an increase in the prevalence of highrisk drinking $(+39.5 \%)$ contrasted against a small decline $(-7.8 \%)$ during the same time-period in 2018/19. This result did not differ significantly by age, but was moderated by sex $(\mathrm{aOR}=1.32,95 \%$ $\mathrm{Cl}=1.09-1.61)$ and social grade $(\mathrm{aOR}=1.48$, $95 \% \mathrm{Cl}=1.21-1.80)$. While increases in high-risk drinking were observed across all groups compared with small declines in 2018/19, there were greater increases among women $(+55.4 \%)$ than men $(+30.7 \%)$ and people from social grades C2DE (+63.9\%) than ABC1 (+29.2\%).

Among high-risk drinkers, lockdown was associated with a significant increase in alcohol reduction attempts $(+75.5 \%)$ relative to the same time-period in $2018 / 19$ $(-7.8 \%)$. This increase was moderated by social grade (aOR $=0.54,95 \% \mathrm{Cl}=0.33-0.87$ ). While substantial increases in the prevalence of alcohol reduction attempts were observed both in social grades $A B C 1$ $(+76.8 \%)$ and $\mathrm{C} 2 \mathrm{DE}(+82.9 \%)$, alcohol reduction attempts had been fairly stable during the same timeperiod in 2018/19 in social grades ABC1 (-9.2\%) but had increased by a similar magnitude in social grades C2DE $(+60.8 \%)$. Thus, the lockdown appeared only to be significantly associated with increased alcohol reduction attempts among high-risk drinkers from social grades ABC1.

Among high-risk drinkers who made an alcohol reduction attempt, lockdown was associated with a decline in use of evidence-based support (-60.0\%) compared with an increase $(+37.2 \%)$ during the same time-period in 2018/19. This change was not statistically significant (possibly due to the small sample sizes; see Table 3 footnote). There was no significant association overall between lockdown and use of remote support by high-risk drinkers, nor evidence of significant moderation by age, sex or social grade.

\section{Sensitivity analyses}

Results of sensitivity analyses excluding data from the month of July are shown in Supporting information, File S4. While effect sizes differed slightly, there were just two notable differences in the pattern of results. First, the moderating effect of social grade on the association between lockdown and alcohol reduction attempts was no longer statistically significant (although the interaction effect size was similar). Secondly, there was 


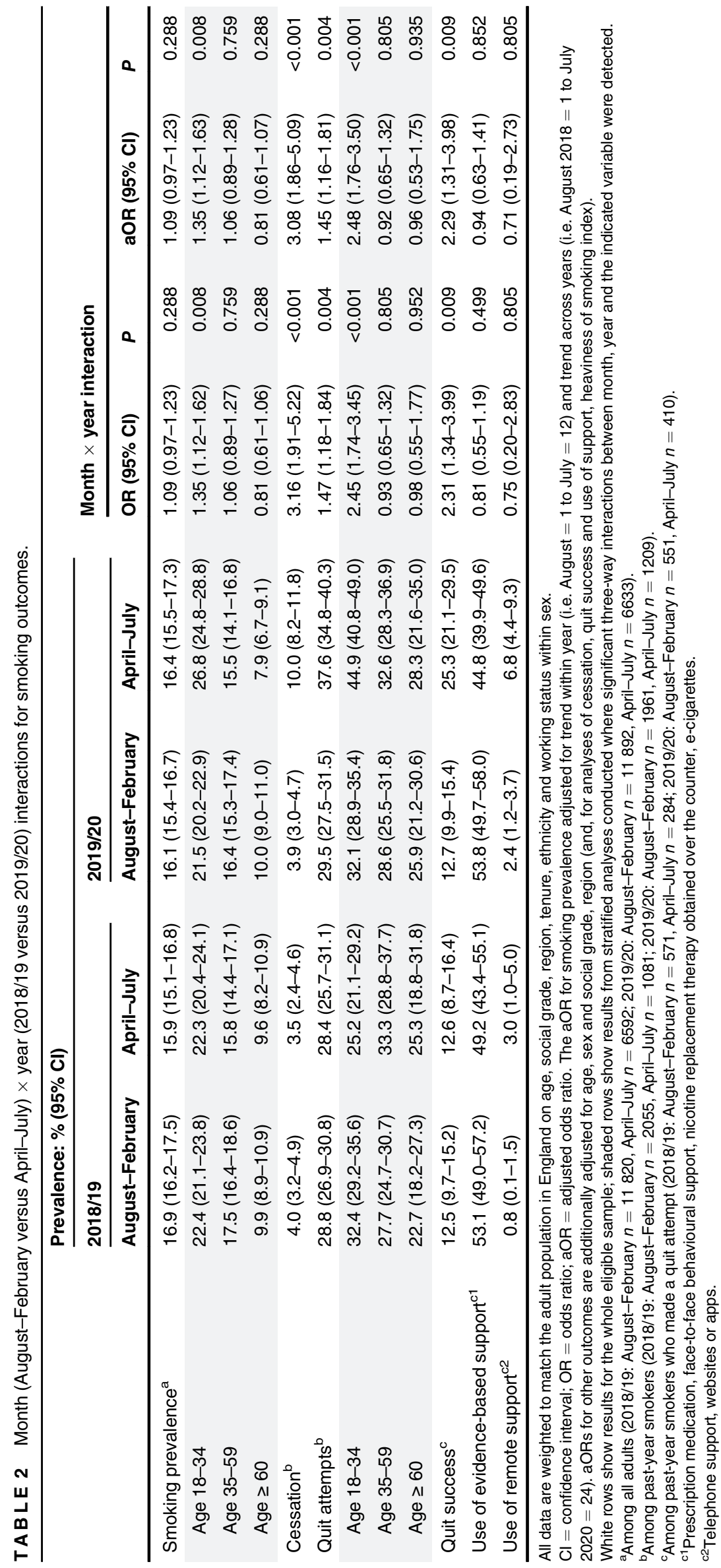




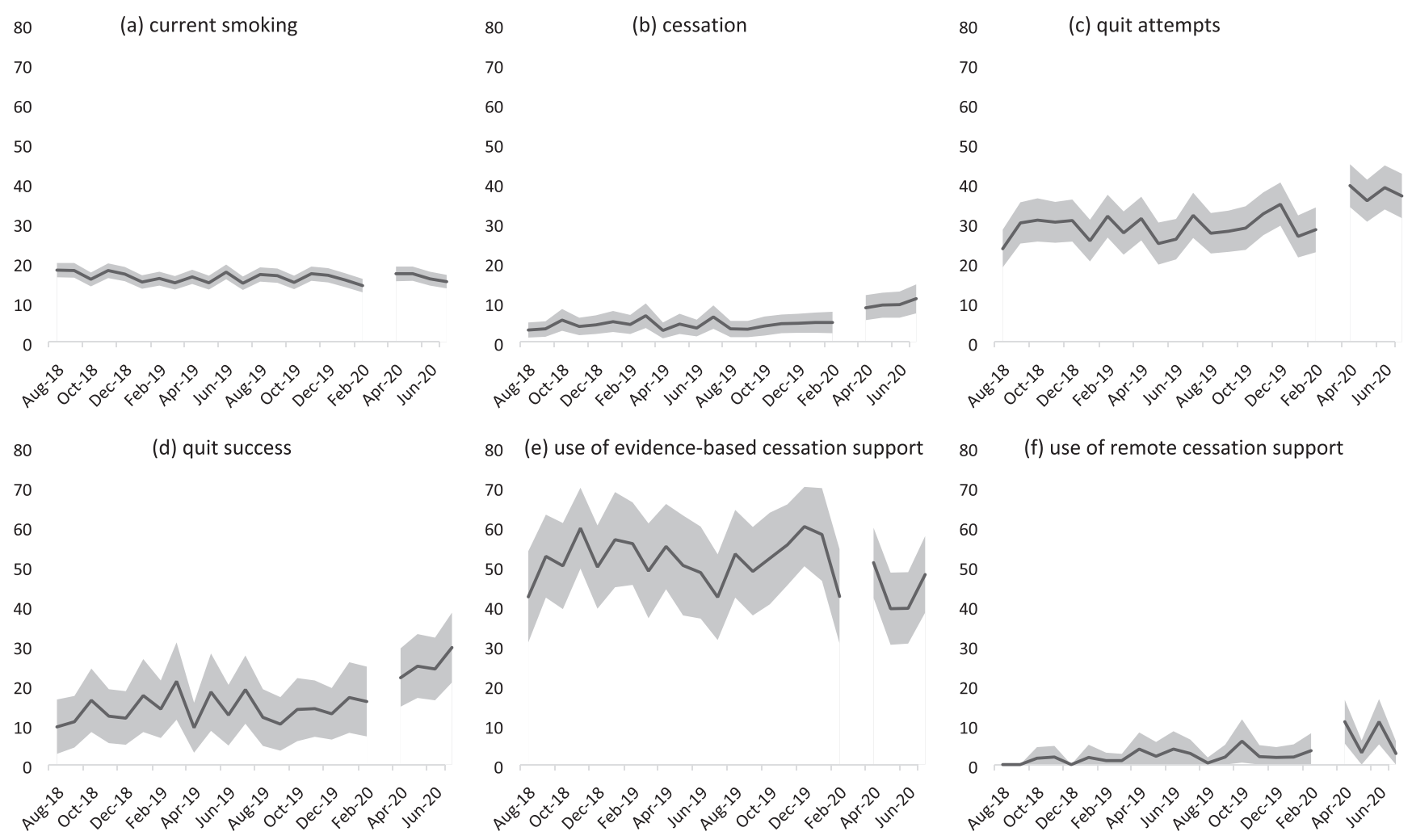

FI G U RE 1 Prevalence of (a) current smoking among all adults; (b) cessation and (c) quit attempts by past-year smokers; and (d) quit success, (e) use of evidence-based cessation support and ( $f$ ) use of remote cessation support by past-year smokers who made a quit attempt in England, August 2018 to July 2020. The break in the line at March 2020 indicates the timing of the COVID-19 lockdown in England (no data were collected this month). The shaded band shows the $95 \%$ confidence interval

evidence of a significant moderating effect of social grade on the association between lockdown and use of remote support for alcohol reduction. Sample sizes were small, but a significant association was observed between lockdown and decreased use of remote support among high-risk drinkers from social grades C2DE $(-48.3 \%)$ compared to an increase in $2018 / 19(+518.2 \%)$.

\section{DISCUSSION}

The first COVID-19 lockdown was associated with significant changes in smoking, drinking and quitting behaviour among adults in England compared with changes throughout the same period a year previously. Smoking prevalence and quit attempts increased comparatively among 18-34-year-olds, but not older groups. Cessation and the success rate of quit attempts also increased comparatively, with no evidence of moderation by age, sex or social grade. Highrisk drinking prevalence increased comparatively across all groups, but particularly pronounced rises were seen in women and people from less advantaged social grades (C2DE). Alcohol reduction attempts increased significantly comparatively among high-risk drinkers from social grades $A B C 1$ but not C2DE, for whom there had been a similar increase in equivalent period a year previously. There was little evidence of significant changes in use of support for smoking cessation or alcohol reduction, although sample sizes were small-particularly when the sample was stratified by age, sex or social grade.

These results build upon and extend our previous analysis of data from the first month of lockdown [12] by covering the full duration of the first lockdown in England (April-July) and exploring the extent to which changes in smoking and drinking outcomes differed by age, sex and social grade. There are two key findings. The first is that the changes in smoking, drinking and quitting we documented in the first month of lockdown appear to have broadly persisted throughout the 4-month lockdown. The only exception concerned changes in use of support, which fluctuated and were not statistically significant. This suggests that increases in high-risk drinking, efforts to reduce alcohol consumption and quit smoking and success in the latter were not short-lived, acute reactions to lockdown. Figures 1 and 2 provide an indication of trends during the lockdown period and are not suggestive of any substantial decay 


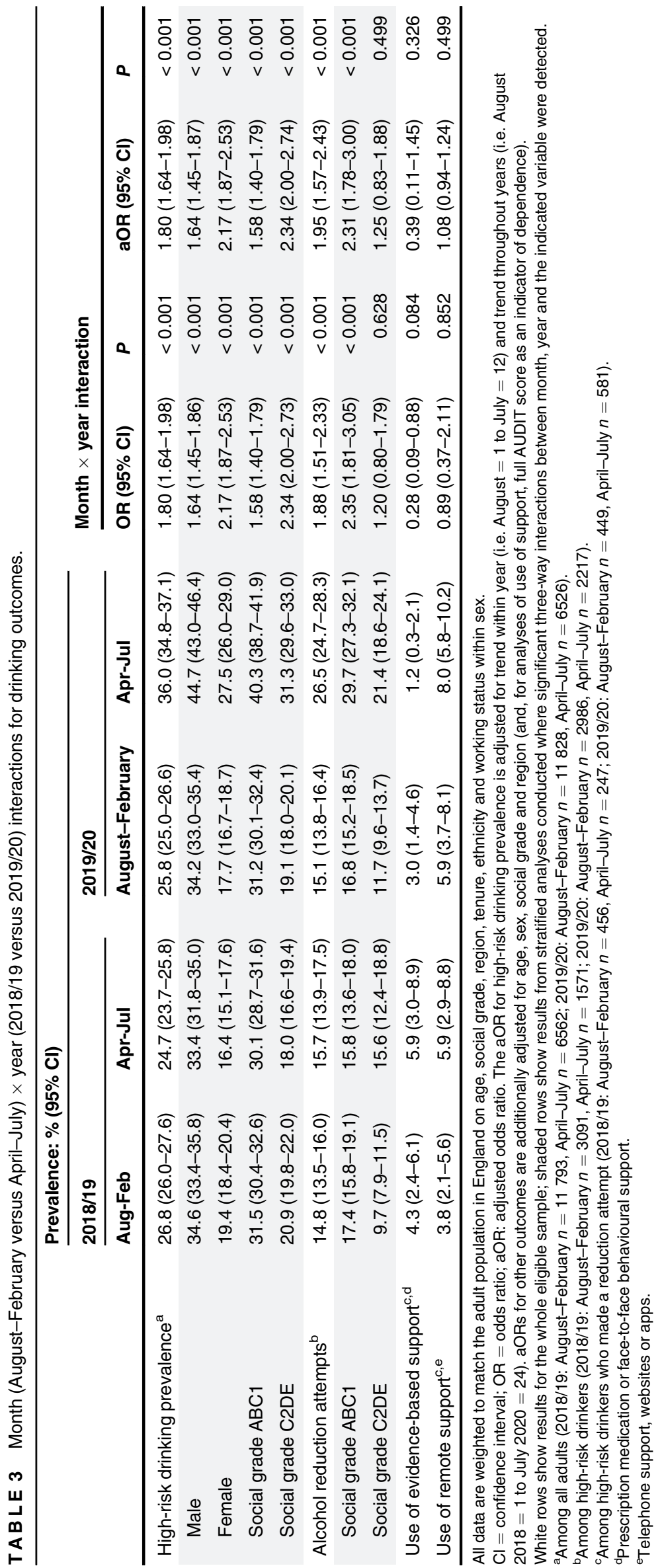




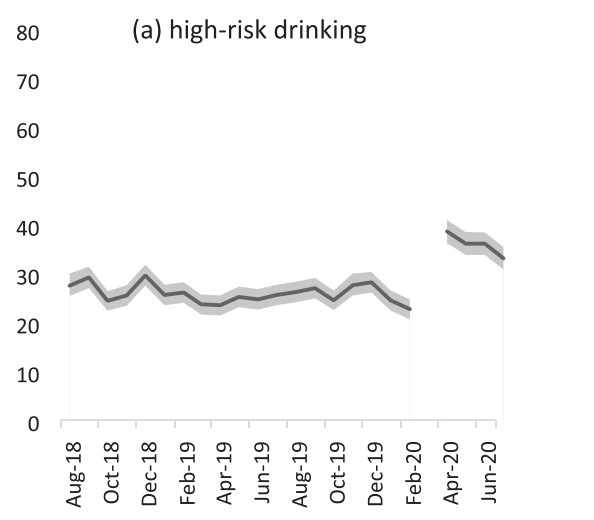

80 (c) use of evidence-based support for alcohol

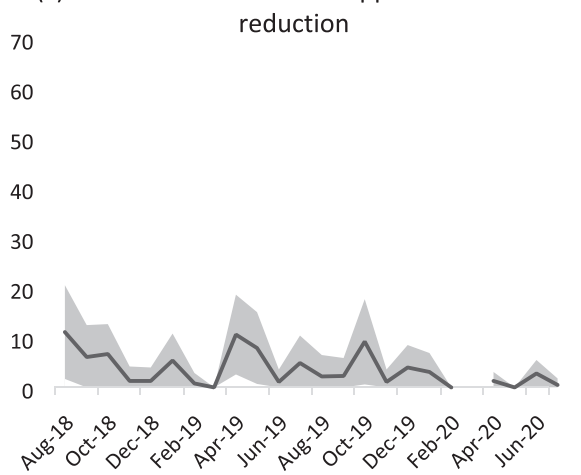

80

(b) alcohol reduction attempts

70

60

50

40

30

20

10

0

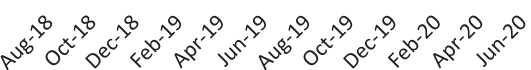

(d) use of remote support for alcohol reduction
50

40

30

20

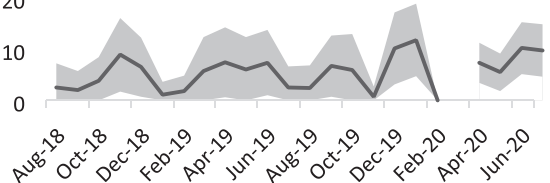

FIG URE 2 Prevalence of (a) high-risk drinking among all adults; (b) reduction attempts by high-risk drinkers; and (c) use of evidence-based support and (d) use of remote support for alcohol reduction by high-risk drinkers who made a reduction attempt in England, August 2019 to July 2020. The break in the line at March 2020 indicates the timing of the COVID-19 lockdown in England (no data were collected this month). The shaded band shows the $95 \%$ confidence interval in our observed effects throughout the 4-month lockdown, with the possible exception of high-risk drinking prevalence. Further analyses of longer-term trends beyond the first lockdown and during subsequent periods of differing COVID-19 restrictions will provide interesting insight into the duration of these changes and the extent to which they recurred during later lockdowns.

The second key finding is that changes in smoking, drinking and quitting behaviours have not occurred equally among all socio-demographic groups. An increase in smoking prevalence during lockdown was only evident among younger adults (aged 18-34 years), with rates relatively stable among older age groups. This might be explained by differential impacts of the pandemic on younger versus older adults: several studies have shown that younger adults report higher levels of pandemic-related stress, say their lives have changed more due to the pandemic, feel more socially isolated and have lower levels of psychological wellbeing $[1,17]$. Many people mistakenly believe that smoking relieves stress $[18,19]$, so those experiencing lockdown-induced stress may have taken up or relapsed to smoking in an effort to cope. Given that older people report being more worried about becoming seriously ill from COVID-19 [20,21], health concerns might have served as a greater deterrent from smoking at older than younger ages. The increase in quit attempts we observed was also concentrated in the younger age group. The apparent discordance between increased prevalence and increased quit attempts among younger adults does not have an obvious explanation and warrants further investigation. It may relate to substantial and unprecedented changes in demography: more than a million people were estimated to have left England during the pandemic [22]. Participant characteristics remained relatively stable from before to during the pandemic, with the exception of the proportion of 18-24-year-olds (see Table 1). If smoking prevalence among the group leaving was lower than the population remaining, then national prevalence estimates could appear to increase despite quitting activity. The pandemic may also have negatively impacted uptake and late relapse (> 1 year), which could lead to increases in prevalence despite the increases in short-term ( $<1$ year) quitting activity that were observed in the current study.

With regard to drinking outcomes, an increase in high-risk drinking prevalence was observed across all socio-demographic groups, but the change was greater among women than men and among adults from social grades $\mathrm{C} 2 \mathrm{DE}$ (less advantaged) than $\mathrm{ABC} 1$ (more advantaged). In addition, a significant increase in alcohol reduction attempts was only observed among highrisk drinkers from social grades $\mathrm{ABC} 1$ : while absolute 
changes from before to during lockdown were similar among social grades, the change in social grades C2DE was comparable with changes during the same time-period in the previous year. The greater increase in high-risk drinking among women than men has been documented in other surveys [23-25] and may reflect stress associated with an exacerbation of gender inequalities: during the pandemic, women have experienced higher rates of job loss and taken on a disproportionately greater share of housework, childcare and home-schooling responsibilities [2,3,26,27]. The greater increase in high-risk drinking among less socioeconomically advantaged groups has not consistently been observed, with a previous survey finding that low income was associated with drinking less than usual during the first few weeks of the lockdown and high income and post-16 qualifications were associated with drinking more [23]. Differences between these results could be due to differences in methodology (i.e. measures used to assess drinking) or timing: the present results cover the full duration of the lockdown and may reflect cumulative effects of the pandemic on less advantaged social grades. The pandemic has worsened socio-economic inequalities [2,4,28], which may have driven the greater increase in high-risk drinking among people from less advantaged social grades and made attempting to reduce alcohol consumption less of a priority.

The present findings have implications for public health. While lockdown restrictions have been necessary to control COVID-19 transmission, they may have adversely affected population health through the increased prevalence of high-risk drinking and increased uptake of or relapse to smoking among younger adults. With greater increases in high-risk drinking among adults from social grades C2DE than $A B C 1$ and increased alcohol reduction attempts among social grades $A B C 1$, but not $C 2 D E$, socio-economic inequalities in health may worsen as a result of lockdown-associated drinking. We note that although increases in high-risk drinking were comparatively smaller among men and social grades $A B C 1$ than women and social grades C2DE, respectively, the former groups had a higher prevalence of high-risk drinking both before and during lockdown. It will be important to monitor the extent to which changes in smoking and drinking during lockdown are sustained during the medium and long term in order to evaluate the full public health impact of the pandemic and help to tailor future harm reduction interventions.

Strengths of this study include the large, representative sample and the broad range of data captured on smoking and drinking behaviour. The repeat crosssectional design with data pre-dating the pandemic was also a strength, as was the comparison of changes from before to during lockdown with data from the previous year, which allowed us to rule out seasonal explanations for changes in our outcomes of interest. There were also several limitations. First, despite the large overall sample, analyses for some of the outcomes (e.g. use of support) were limited to relatively small numbers of participants (i.e. smokers/high-risk drinkers who had made a quit/reduction attempt). This resulted in estimates with wide confidence intervals and limited statistical power to detect significant interactions with age, sex or social grade. As such, we emphasize the need to interpret results as providing no evidence of differences between these groups, rather than evidence of no differences. In particular, our finding that smoking quit success increased during lockdown in the absence of evidence of an increase in use of evidence-based support should not be interpreted as these cessation aids not contributing to the change. With lockdown dramatically changing the way people were able to live their lives, it is plausible that other factors besides the use of support could explain the observed increase in quit success; for example, a reduction in socializing meant that many 'social' smokers simply were not in the circumstances that they would normally associate with smoking, hence quit attempts might have been more likely to succeed. Secondly, it is possible that the change in modality of data collection from face-to-face (before lockdown) to telephone interviews (during lockdown) may have contributed to some of the changes in smoking and drinking behaviour we observed. However, the diagnostic analyses we undertook comparing the face-to-face and telephone data, combined with previous studies showing a high degree of comparability between face-to-face and telephone interviews $[29,30]$, suggest that it is reasonable to compare data collected via the two methods. Finally, we did not model changes within the lockdown period, so our analyses cannot conclusively tell us whether immediate changes after lockdown was implemented were sustained, decayed or even increased during the 4 months of lockdown. We plan to conduct more sophisticated interrupted time-series modelling when sufficient post-lockdown data points are available. However, we provide descriptive data on monthly changes in each outcome in Figures 1 and 2 to supplement our primary analyses of aggregated data.

In conclusion, the first COVID-19 lockdown in England in March-July 2020 was associated with increased smoking prevalence among younger adults and an increased prevalence of high-risk drinking among all socio-demographic groups. Smoking cessation activity also increased: more younger smokers made quit attempts during lockdown and more smokers quit successfully. However, socio-economic disparities in patterns of drinking behaviour were evident: high-risk drinking increased by more among women and those from less advantaged social grades, but the rate of alcohol reduction attempts increased only among the more advantaged social grades. 


\section{CONFLICT OF INTEREST}

J.B. and E.B. have received unrestricted research funding from Pfizer, who manufacture smoking cessation medications. All authors declare no financial links with tobacco companies or e-cigarette manufacturers or their representatives.

\section{ACKNOWLEDGEMENTS}

Data collection for the Smoking and Alcohol Toolkit Studies and S.J. and E.B.'s salaries were supported by Cancer Research UK (C1417/A22962).

\section{AUTHOR CONTRIBUTIONS}

Sarah Jackson: Conceptualization; formal analysis; investigation; methodology; visualization. Emma Beard: Conceptualization; investigation; methodology. Colin Angus: Conceptualization; investigation; methodology. Matt Field: Conceptualization; investigation; methodology. Jamie Brown: Conceptualization; data curation; funding acquisition; investigation; methodology; project administration; supervision.

\section{ORCID}

Sarah E. Jackson (1) https://orcid.org/0000-0001-56586168

Emma Beard (1) https://orcid.org/0000-0001-8586-1261 Colin Angus (10) https://orcid.org/0000-0003-0529-4135 Matt Field (1) https://orcid.org/0000-0002-7790-5559 Jamie Brown (10 https://orcid.org/0000-0002-2797-5428

\section{REFERENCES}

1. Birditt KS, Turkelson A, Fingerman KL, Polenick CA, Oya A. Age differences in stress, life changes, and social ties during the COVID-19 pandemic: implications for psychological well-being. Gerontologist [internet] 2020; 61: 205-216. https://doi.org/10. 1093/geront/gnaa204. (accessed: September 12020 ).

2. Adams-Prassl A, Boneva T, Golin M, Rauh C. Inequality in the impact of the coronavirus shock: evidence from real time surveys. J Public Econ. 2020;189:104245.

3. Landivar LC, Ruppanner L, Scarborough WJ, Collins C. Early signs indicate that COVID-19 is exacerbating gender inequality in the labor force. Socius. 2020;6:2378023120947997.

4. Blundell R, Dias MC, Joyce R, Xu X. COVID-19 and inequalities. Fisc Stud. 2020;41:291-319.

5. Official UK Coronavirus Dashboard [internet]. 2021 [cited 2021 May 11]. Available at: https://coronavirus.data.gov.uk/ details/deaths. (accessed: September 12020 ).

6. Jit $\mathrm{M}$, Jombart $\mathrm{T}$, Nightingale ES, et al. Estimating number of cases and spread of coronavirus disease (COVID-19) using critical care admissions, United Kingdom, February to March 2020. Eurosurveillance. 2020;25:2000632.

7. Department of Health and Social Care. Coronavirus (COVID-19) Action Plan [internet]. GOV.UK. 2020 [cited 2020 May 14]. Available at: https://www.gov.uk/government/publications/ coronavirus-action-plan. (accessed: September 1 2020).

8. Institute for Government. Timeline of UK coronavirus lockdowns, March 2020 to March 2021 [internet]. 2021 [cited 2021 May 11]. Available at: https://www.instituteforgovernment.org.uk/sites/ default/files/timeline-lockdown-web.pdf. (accessed: September 12020).

9. Prime Minister's statement on coronavirus (COVID-19): 23 March 2020 [internet]. GOV.UK. 2020 [cited 2021 May 11]
Available at: https://www.gov.uk/government/speeches/pmaddress-to-the-nation-on-coronavirus-23-march-2020. (accessed: September 12020 ).

10. Fidler JA, Shahab L, West O, et al. 'The Smoking Toolkit Study': a national study of smoking and smoking cessation in England. BMC Public Health. 2011;11:479.

11. Beard E, Brown J, West R, Acton C, Brennan A, Drummond C et al. Protocol for a national monthly survey of alcohol use in England with 6-month follow-up: 'the Alcohol Toolkit Study'. BMC Public Health 2015;15:230.

12. Jackson SE, Garnett C, Shahab L, Oldham M, Brown J. Association of the COVID-19 lockdown with smoking, drinking, and attempts to quit in England: an analysis of 2019-2020 data. Addiction 2021; 116; 1233-1244 [internet] [cited 2020 Nov 11]. Available at: https://doi.org/10.1111/add.15295. (accessed: September 1 2020).

13. Jackson SE, Beard E, Kujawski B, et al. Comparison of trends in self-reported cigarette consumption and sales in England, 2011 to 2018. JAMA Netw Open. 2019;2:e1910161-1.

14. Babor TF, Higgins-Biddle JC, Saunders JB, Monteiro MG. AUDIT: the alcohol use disorders identification test: guidelines for use in primary health care. Geneva: World Health Organization; 2001.

15. Heatherton TF, Kozlowski LT, Frecker RC, Rickert W, Robinson J. Measuring the heaviness of smoking: using selfreported time to the first cigarette of the day and number of cigarettes smoked per day. Br J Addict. 1989;84:791-800.

16. Benjamini $Y$, Hochberg $Y$. Controlling the false discovery rate: a practical and powerful approach to multiple testing. J R Stat Soc Ser B Methodol. 1995;289-300.

17. Nwachukwu I, Nkire N, Shalaby R, Hrabok M, Vuong W, Gusnowski A et al. COVID-19 pandemic: age-related differences in measures of stress, anxiety and depression in Canada. Int $\mathrm{J}$ Environ Res Public Health 2021; 17 [internet]. 2020:6366. [cited 2021 Jan 20]. https://www.ncbi.nlm.nih.gov/pmc/articles/ PMC7503671/. (accessed: September 12020 ).

18. Fidler JA, West R. Self-perceived smoking motives and their correlates in a general population sample. Nicotine Tob Res Off J Soc Res Nicotine Tob. 2009;11:1182-8.

19. Parrott AC. Does cigarette smoking cause stress? Am Psychol. 1999:54:817-20.

20. Fancourt D, Bu F, Mak HW, Steptoe A. Covid-19 Social Study: Results Release 1 [internet]. 2020 [cited 2020 Feb 5]. Available at: https://746a1e8d-7231-4b96-9bc2-88b2eb5c4964.filesusr. com/ugd/3d9db5_a82c3a15441f4687a0114efc78307e80.pdf. (accessed: September 12020 ).

21. Maxfield M, Pituch KA. COVID-19 worry, mental health indicators, and preparedness for future care needs across the adult lifespan. Aging Ment Health. 2021;75:1273-80.

22. Economic Statistics Centre of Excellence. Estimating the UK population during the pandemic [internet]. ESCoE. 2021 [cited 2021 Jan 26]. Available at: https://www.escoe.ac.uk/estimatingthe-uk-population-during-the-pandemic/. (accessed: September $12020)$.

23. Garnett C, Jackson S, Oldham M, Brown J, Steptoe A Fancourt D. Factors associated with drinking behaviour during COVID-19 social distancing and lockdown among adults in the UK. Drug Alcohol Depend. 2021;219:108461.

24. YouGov. Daily Question: Are you drinking more or less alcohol than you normally would? [internet]. 2020. Available at: https:// yougov.co.uk/topics/food/survey-results/daily/2020/03/24/0d9bc/ 3. (accessed: September 1 2020).

25. Portman Group. YouGov/Portman Group survey on alcohol consumption during the COVID-19 lockdown [internet]. 2020. Available at: https://www.portmangroup.org.uk/wp-content/ uploads/2020/06/YouGov-Portman-Group-survey-on-alcoholconsumption-during-the-COVID-19-lockdown-2.pdf. (accessed: September 1 2020) 
26. Collins C, Landivar LC, Ruppanner L, Scarborough WJ. COVID-19 and the gender gap in work hours. Gend Work Organ 2020 [internet]. [cited 2021 Jan 20]. 101-112. https://doi. org/10.1111/gwao.12506. (accessed: September 1 2020).

27. Farre L, Fawaz Y, Gonzalez L, Graves J. How the Covid-19 Lockdown Affected Gender Inequality in Paid and Unpaid Work in Spain [internet]. Rochester, NY: Social Science Research Network; 2020 Jul [cited 2021 Jan 20]. Report no.: ID 3643198. Available at: https://papers.ssrn.com/abstract= 3643198. (accessed: September 1 2020).

28. van Dorn A, Cooney RE, Sabin ML. COVID-19 exacerbating inequalities in the US. Lancet. 2020;395:1243-4.

29. Aziz MA, Kenford S. Comparability of telephone and face-to-face interviews in assessing patients with posttraumatic stress disorder. J Psychiatr Pract. 2004;10:307-13.

30. Midanik LT, Hines AM, Greenfield TK, Rogers JD. Face-to-face versus telephone interviews: using cognitive methods to assess alcohol survey questions. Contemp Drug Probl. 1999;26: 673-93.

\section{SUPPORTING INFORMATION}

Additional supporting information may be found in the online version of the article at the publisher's website.

How to cite this article: Jackson SE, Beard E, Angus $\mathrm{C}$, Field M, Brown J. Moderators of changes in smoking, drinking and quitting behaviour associated with the first COVID-19 lockdown in England. Addiction. 2021;1-13. https://doi.org/10.1111/add.15656 